SCIENTIFIC LETTER

\title{
Vascular endothelial growth factor (VEGF) plasma concentrations in coronary artery disease
}

\author{
H F Alber, M Frick, J Dulak, J Dörler, R-H Zwick, W Dichtl, O Pachinger, F Weidinger
}

Heart 2005;91:365-366. doi: 10.1136/hrt.2003.021311

$\mathrm{V}$ ascular endothelial growth factor (VEGF) has been associated with atherosclerosis progression and lesion destabilisation. Despite a beneficial effect of local VEGF administration in myocardial and peripheral ischaemia, recent evidence suggests a pro-atherosclerotic role of $\mathrm{VEGF}^{2}$ through its ability to enhance plaque inflammatory infiltration and neovascularisation.

Despite these results, coming mostly from animal studies, there have been few investigations on the relation between VEGF and human coronary artery disease (CAD). These studies yielded conflicting results regarding VEGF concentrations and gene expression in CAD patients compared to controls. ${ }^{3}{ }^{4}$ To elucidate the association of VEGF and CAD further, we performed a prospective study in consecutive patients with chest pain undergoing coronary angiography to compare the coronary status with VEGF plasma concentrations.

\section{MATERIALS AND METHODS}

Written informed consent was obtained from 178 patients. Plasma was collected from a femoral artery access immediately before diagnostic coronary angiography. Exclusion criteria were prior myocardial infarction $(<1$ month before inclusion), tumour disease, peripheral arterial occlusive disease, ejection fraction $<30 \%$, acute infections, chronic rheumatoid diseases, and chronic obstructive pulmonary disease. Prior statin use was defined as statin treatment for more than 10 days. VEGF plasma concentrations were determined by enzyme linked immunosorbent assay (ELISA, R\&D System, Abingdon, UK).

Coronary angiograms were scored visually by a blinded observer: a severity score (0-3) defined the number of vessels with a luminal stenosis $\geqslant 50 \%$ (for right, left anterior descending, and circumflex arteries). The severity and extent of CAD was graded using a modified Gensini score. The most severe stenosis in each of the three coronaries and in the left main was graded from 0 to 6 (0, no stenosis; $1,1-29 \%$ stenosis; 2, 30-49\% stenosis; 3, 50-69\% stenosis; 4, 70-89\% stenosis; $5,90-99 \% ; 6,100 \%$ occlusion) and summed to yield a score of $0-24$. Seventeen angiograms were excluded because of missing of at least two orthogonal views of all coronaries.

The angiogenic activity of patient plasma was determined in a matrigel matrix. Human umbilical vein endothelial cells (HUVEC), seeded on matrigel, were incubated without growth factors and supplemented with $10 \mu \mathrm{l}$ of plasma from 42 randomly selected patients, and the number of HUVEC tubes formed was counted.

Values are expressed as mean (SD). Kruskal-Wallis-H test or Mann-Whitney-U test were used for non-parametric comparisons and ANOVA with post hoc Scheffe test or $t$ test were used for parametric comparisons. Correlations were evaluated with Pearson's or Spearman's correlation coefficients. A probability value of $\mathrm{p}<0.05$ was considered significant.

\section{RESULTS}

There was no difference in VEGF concentrations in patients without CAD (score 0) compared to those with CAD (score 1$3)$, when analysed in regard to the severity score (fig lA). High density lipoprotein (HDL) cholesterol was lower, and triglycerides were higher in patients with triple vessel disease compared to patients with smooth coronaries (ANOVA, $\mathrm{p}<0.03$, post hoc $\mathrm{p}<0.05)$ and both variables correlated with the severity score $(r=-0.23, \mathrm{p}=0.008 ; r=0.25$, $\mathrm{p}<0.005$, respectively). Other laboratory parameters and risk factors were not different. As the severity and the modified Gensini score were correlated $(r=0.89$, $\mathrm{p}<0.001$ ), the above mentioned results did not substantially change when VEGF was related to the latter. The adjustment to leucocyte and thrombocyte counts, which correlated weakly with VEGF $(r=0.17, \mathrm{p}<0.03$ and $r=0.36$, $\mathrm{p}<0.001$ ), did not change the results.

As statin treatment decreases VEGF, ${ }^{5}$ patients were divided into two groups according to prior statin use. Statin treated patients had lower VEGF (24.1 (20.2) v 46.2 (54.6) pg/ml; $\mathrm{p}<0.05$ ) (fig $1 \mathrm{~B}$ ) and lower low density lipoprotein (LDL) cholesterol (125.6 (40.0) v 144.7 (39.5) mg/dl; p < 0.02 ) concentrations than patients without statin treatment. There were more diabetic, hypertensive, and smoking patients in the untreated group.

Among 42 randomly selected patients, eight were undergoing statin treatment. The plasma from these patients induced the formation of 6.3 (3.1) tubes and the plasma from untreated patients stimulated 9.1 (4.9) tubes in the matrigel assay $(\mathrm{p}=0.12)$ (fig $\mathrm{lC})$.

\section{DISCUSSION}

This study shows that VEGF plasma concentrations do not correlate with the severity and extent of CAD. Statin treated patients have lower VEGF concentrations compared to untreated patients.

Although VEGF has been considered for angiogenic gene therapy in patients with $\mathrm{CAD},{ }^{1}$ data on circulating and tissue VEGF concentrations in patients with documented CAD are scarce. In our data VEGF appears to be similar in CAD patients and patients without diseased coronaries. In fact, VEGF has recently been implicated to promote atherosclerosis by inducing plaque neovascularisation. ${ }^{2}$

To our knowledge, this is the first study comparing VEGF concentrations with angiographically documented CAD in a large patient population. In accordance with our data, Fleisch and colleagues ${ }^{4}$ observed no substantial differences in VEGF in patients with various extents of CAD. In contrast, increased VEGF concentrations in CAD patients were reported by Blann and colleagues. ${ }^{3}$ This discrepancy is probably because of different patient populations. Firstly, CAD patients in the latter study had higher cholesterol

Abbreviations: CAD, coronary artery disease; HUVEC, human umbilical vein endothelial cells; VEGF, vascular endothelial growth factor 


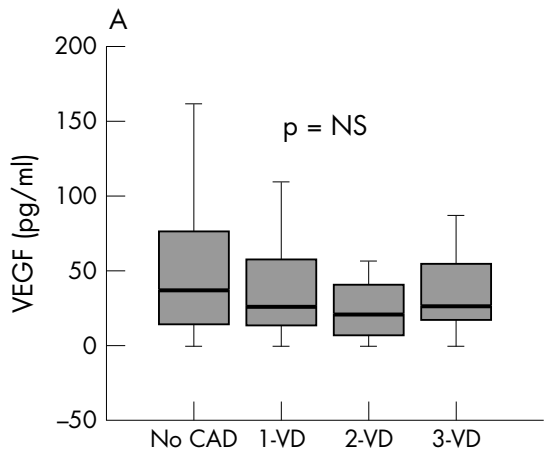

\section{C}

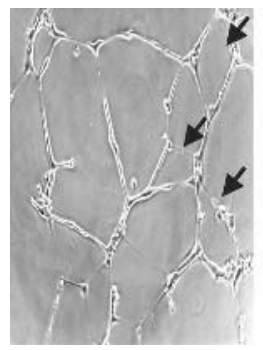

No statin treatment

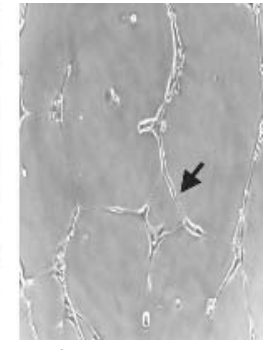

B

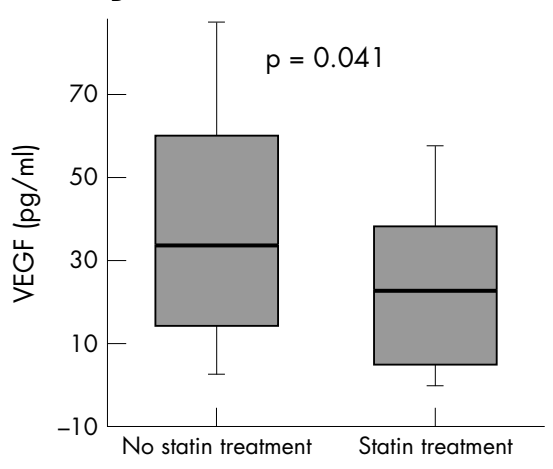

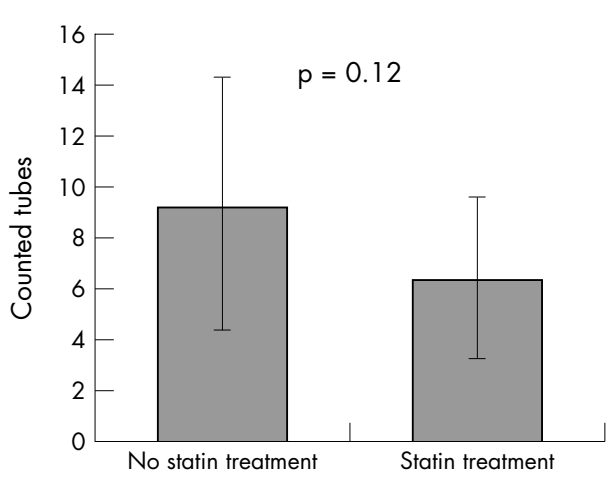

Figure 1 (A) VEGF plasma concentrations are similar in patients with different severity scores of CAD and in patients with smooth coronaries ( $p=$ not significant). (B) VEGF plasma concentrations are lower in patients $(\mathrm{n}=30)$ under statin treatment compared to patients without statin treatment $(\mathrm{n}=148, \mathrm{p}<0.05)$. Box plots display medians, 25th and 75th centiles (boxes), and 10th and 90th centiles (whiskers). (C) Pictures show HUVECs incubated in a matrigel assay with $10 \mu \mathrm{l}$ of plasma collected from patients without (left) and with (right) statin treatment. Bars show mean value (SD) from counted tubes by two independent observers. Plasma collected from statin treated patients tended to stimulate less tube formation than plasma from untreated patients (arrows indicate tubes, $p=0.12$ ). concentrations compared to controls. Cholesterol may stimulate VEGF production and hyperlipidaemic patients have higher VEGF concentrations compared to healthy subjects. ${ }^{5}$ Secondly, patients with recent acute myocardial infarction were excluded from our study, but were selectively included in the study by Blann and colleagues. Elevated VEGF concentrations were reported after acute myocardial infarction. Thirdly, our controls were referred for coronary angiography because of chest pain, whereas controls in the study by Blann and colleagues ${ }^{3}$ consisted of healthy volunteers.

We confirmed our previous results ${ }^{5}$ demonstrating a VEGF lowering effect of statins in this larger patient population. Although there was no significant influence on tube formation in a matrigel assay, the observed inhibitory tendency of plasma from statin treated patients supports an anti-angiogenic effect of statins.

In conclusion, VEGF plasma concentrations do not correlate with the presence, severity, and extent of CAD. Statin treatment was associated with lower VEGF plasma concentrations. Further studies are required to examine the effects of statins on VEGF and on plaque neovascularisation in humans.

\section{ACKNOWLEDGEMENTS}

Dr J Dulak was the recipient of the fellowship of Austrian Society for Cardiology (1999-2001). The study was supported in part by the
Polish-Austrian Collaboration Project. We thank Eva-Maria Mair for technical support.

\section{Authors' affiliations}

H F Alber, M Frick, J Dulak, J Dörler, R-H Zwick, W Dichtl, O Pachinger, F Weidinger, Department of Cardiology, University of Innsbruck, Innsbruck, Austria

Correspondence to: Dr Franz Weidinger, Department of Cardiology, University of Innsbruck, A-6020 Innsbruck, Austria; f.weidinger@uibk. ac.at

Accepted 15 April 2004

\section{REFERENCES}

1 Losordo DW, Vale PR, Symes JF, et al. Gene therapy for myocardial angiogenesis : initial clinical results with direct myocardial injection of phVEGF $_{165}$ as sole therapy for myocardial ischemia. Circulation 1998;98:2800-4

2 Celletti FL, Waugh JM, Amabile PG, et al. Vascular endothelial growth factor enhances atherosclerotic plaque progression. Nat Med 2001 7:425-9.

3 Blann AD, Belgore AM, McCollum CN, et al. Vascular endothelial growth factor and its receptor, Flt-1, in the plasma of patients with coronary or peripheral atherosclerosis, or type II diabetes. Clin Sci 2002;102:187-94.

4 Fleisch $M$, Billinger $M$, Eberli FR, et al. Physiologically assessed coronary collateral flow and intracoronary growth factor concentrations in patients with 1- to 3-vessel coronary artery disease. Circulation 1999;100:1945-50.

5 Alber HF, Dulak J, Frick M, et al. Atorvastatin decreases vascular endothelial growth factor in patients with coronary artery disease. J Am Coll Cardiol 2002;39:1951-5. 